Boletín de la Sociedad Geológica Mexicana

VOLUMEN 62, NÚM. 2, 2010, P. 221-231

\title{
Tafofacies e icnofacies de los niveles maastrichtianos-danianos correspondientes a la Formación Yacoraite, Cordillera Oriental de la provincia de Jujuy, Argentina
}

\author{
Carlos A. Cónsole Gonella ${ }^{1, *}$, Florencio G. Aceñolaza ${ }^{1}$ \\ ${ }^{1}$ Instituto Superior de Correlación Geológica (INSUGEO - CONICET), Universidad Nacional de Tucumán. Miguel Lillo 205, San \\ Miguel de Tucumán (4000), Argentina. \\ *carlos_console@yahoo.com.ar
}

\begin{abstract}
Resumen
Presentamos en este trabajo la caracterización tafofacial e icnofacial de los niveles sedimentarios correspondientes a la Formación Yacoraite (Maastrichtiano-Daniano), en el área de Maimará, Departamento Tilcara, Cordillera Oriental de la Provincia de Jujuy, noroeste argentino. Con este propósito en el área estudiada se analizaron dos perfiles estratigráficos de detalle. Como resultado se ha determinado una tafofacies G1, correspondiente a una concentración monotípica, poliespecífica de gasterópodos fragmentados, caracterizando proximidad a la costa y alta energía por acción del oleaje, y una tafofacies P1, correspondiente a una concentración de peces retrabajados, monotípica y poliespecífica en estructuras sedimentarias con estratificación entrecruzada tipo monticular (hummoky), lo que indicaría la acción de tempestitas proximales. El análisis de los icnogéneros dominantes Skolithos isp., Arenicolites isp., Diplocraterion isp. y Thalassinoides isp. permitió definir a la icnofacies arquetípica de Skolithos, propia de ambientes de alta energía y buena disponibilidad de nutrientes. Se destaca también el hallazgo de huellas tridactilares asignables a la subclase de aves Enanthiornithes. La interpretación de los resultados de las caracterizaciones tafofacial e icnofacial, y su correlación con las litofacies sedimentarias, permiten la determinación de un ambiente litoral, salobre, somero, con buena oxigenación, alta energía y disponibilidad de nutrientes, dominado por el oleaje y sedimentación episódica breve producto de eventos de tormentas..
\end{abstract}

Palabras clave: Tafofacies, Icnofacies, Argentina, Formación Yacoraite, Maastrichtiano-Daniano.

\begin{abstract}
In this paper we present the taphofacies and ichnofacies characterization of the sedimentary horizons in the Yacoraite Formation (Maastrichtian-Danian) in the environs of Maimará, Tilcara district, Cordillera Oriental of Jujuy province in northwestern Argentina. With this purpose in mind, two detailed stratigraphic sections were analyzed in the study area. As a result we determined a G1 taphofacies, corresponding to a monotypic and polyspecific concentration of fragmented gastropods, indicating proximity to the coast and highenergy wave action, and a P1 taphofacies, corresponding to a monotypic and polyspecific reworked fish concentration, preserved in hummocky cross-stratification structures, indicating the action of proximal tempestites. The analysis of dominant Skolithos isp., Arenicolites isp., Diplocraterion isp. and Thalassinoides isp. ichnogenus allowed us to define the archetypal Skolithos ichnofacies, typical of high-energy environments and good availability of nutrients. Also, we emphasize the record of tridactil footprints, which are assigned to the subclass of birds Enanthiornithes. The taphofacies and ichnofacies analysis, and its correlation with the sedimentary lithofacies, allow us to define a high-energy shallow depositional environment, mixed, with good oxygenation, and nutrient availability, dominated by waves and brief episodic sedimentation as a product of storm events.
\end{abstract}

Keywords: Taphofacies, Ichnofacies, Argentina, Yacoraite Formation, Maastrichtian-Danian. 


\section{Introducción}

La tafonomía es una disciplina que ha posibilitado la interpretación paleoambiental de secuencias con abundante contenido fosilífero (Lazo, 2006). En este sentido, nuestro conocimiento sobre la resolución temporal y las limitaciones paleoecológicas ofrecidas por los datos dados por aquellas ricas asociaciones fósiles marítimas son ahora más exactas que en el pasado, favoreciendo interpretaciones más precisas y fieles (Bender Kotzian y Simoes, 2006). Si bien la icnología se ocupa de evidencias del comportamiento, sus deducciones no solo informan acerca de aspectos etológicos, sino también de numerosos otros sobre sedimentología y biología (Frey y Seilacher, 1980). La Formación Yacoraite (Maastrichtiano-Daniano) es una de las unidades de mayor extensión lateral en el norte argentino, y constituye un excelente caso de estudio tafonómico e icnológico. Su abundante contenido fósil ha sido revisado en numerosas contribuciones. En particular se han reconocido restos fósiles de peces (Arratia y Cione, 1996; Cione, 1977; Cione y Pereira, 1985; Cione et al., 1985) y reptiles (Gasparini y Buffetaut, 1980), gasterópodos (Bonarelli, 1927; Cónsole Gonella y Aceñolaza, 2008, Cónsole Gonella et al., 2009), ostrácodos, pelecípodos, escasos miliólidos, carófitas y otras algas, restos vegetales y palinomorfos, además de huellas de dinosaurios y de aves (Alonso, 1980; Alonso y Marquillas, 1986 ; Marquillas et al., 2005, 2007; Marquillas y Salfity, 1994). En este sentido destacamos para la localidad de Maimará nuestro registro de huellas tridactilares asignables a la subclase de aves Enanthiornithes (Walker, 1981) y la presencia de huellas de dinosaurio bípedos y tridáctilos (Alonso, 2007).

De igual manera, su icnología ha demostrado una interesante diversidad, Cónsole Gonella et al. (2009) identificaron a la icnofacies de Glossifungites, siendo notoria la predominancia en estos niveles de los icnogéneros Skolithos, Gastrochaenolites y Trypanites.

El enfoque de este aporte radica en la integración y correlación de datos tafonómicos e icnológicos. Este tipo de investigaciones han sido exitosamente encaradas (Bressan y Palma, 2008; Bromley, 1990, 1996; Davies et al., 2007; Donovan y Jagt, 2004; Kietzmann y Palma 2009; Lazo, 2006; entre otros) permitiendo obtener datos geológicos precisos, en particular aquellos vinculados con aspectos batimétricos de las cuencas analizadas.

Con esta finalidad, se relevaron dos perfiles de detalle en el área de Maimará, Departamento Tilcara, Jujuy, Argentina (Figura 1), describiéndose las litofacies, tafofacies e icnofacies aflorantes.

\section{Marco Geológico}

La Formación Yacoraite es una de las unidades que integran el Grupo Salta (Turner, 1959), que a su vez se divide en tres subgrupos: Pirgua (Reyes y Salfity, 1973), que corresponde a los bancos rojos basales; Balbuena (Moreno, 1970), constituido por areniscas, calizas y pelitas; y Santa Bárbara (Moreno, 1970), formado por pelitas polícromas con calizas y areniscas. Este grupo ha sido definido como una cuenca de tipo rift, cuyos depósitos de $5000 \mathrm{~m}$ de espesor se acumularon entre el Cretácico Inferior-Eoceno y el Eoceno, por lo que la sedimentación abarca la transición cretácico-cenozoica (Marquillas et al., 2003).

La unidad más característica del Subgrupo Balbuena (Moreno, 1970) es la Formación Yacoraite. Se ha definido a la "Cuenca Yacoraite" como una cuenca carbonática restringida, somera y extensa, de posición intracontinental, alejada de las influencias directas y permanentes del mar abierto y por lo tanto con caracteres que le son propios

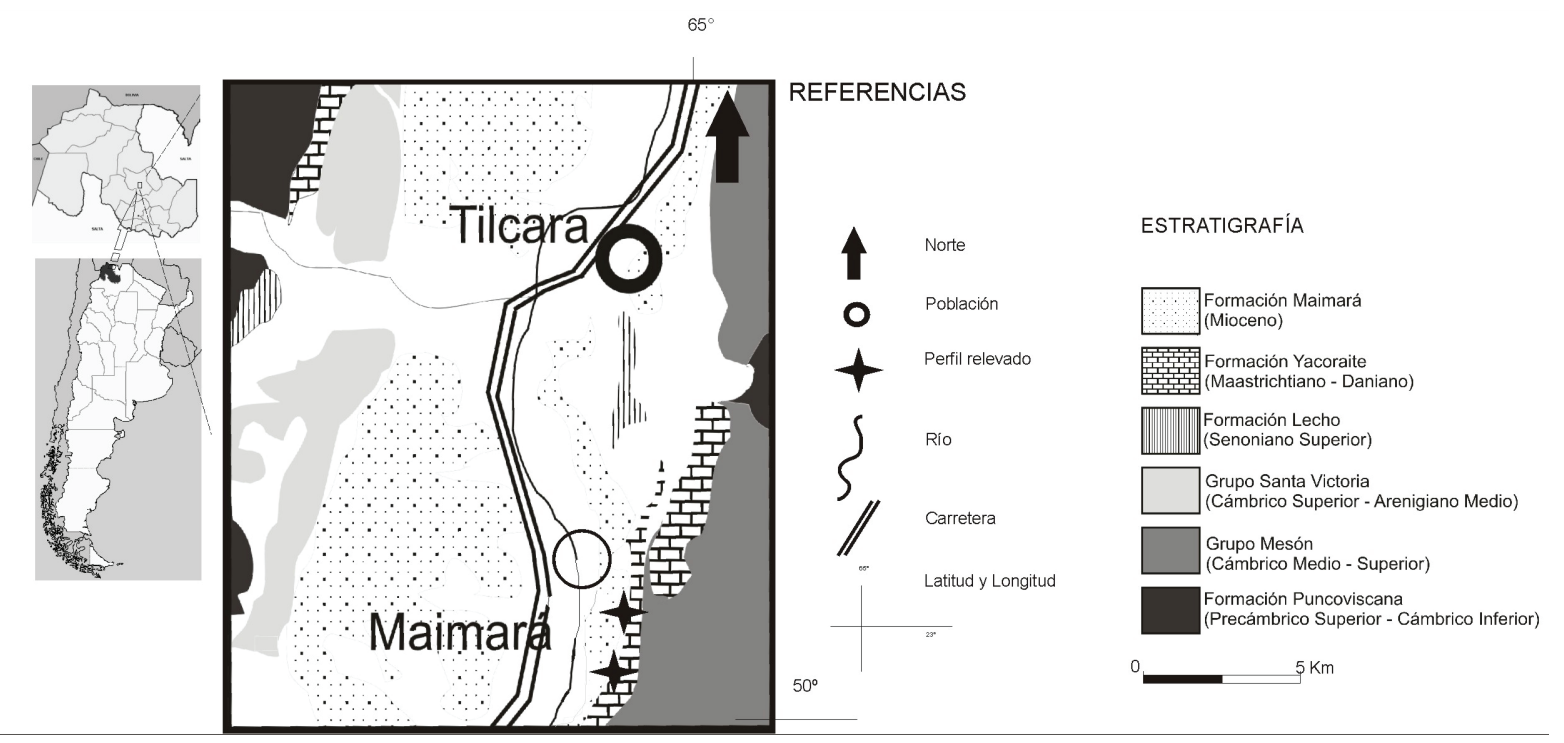

Figura 1: Mapa geológico del área de estudio. 
(Marquillas, 1985). Está ampliamente distribuida en el noroeste argentino en una serie de fosas o subcuencas: Tres Cruces, Lomas de Olmedo, Metán, Alemanía, Cerro Hermoso, El Rey y El Charco o Sey.

\section{Metodología}

Los perfiles relevados (Figura 2) fueron analizados con la finalidad de agrupar a las secuencias estratigráficas originales en facies. El criterio de descripción de litofacies e icnofacies fue modificado de Lazo (2006). Las litofacies se definieron en función de la litología, textura, estructuras sedimentarias, geometría, contactos, empaquetamiento, color y espesor. Cada facies fue denominada utilizando una letra mayúscula correspondiente a su litología y una o dos letras minúsculas acompañantes que expresan las estructuras y/o composición de las secuencias descritas. Los atributos o índices tafonómicos, modificados de Parsons y Brett (1991) y Kowalewski et al. (1994), fueron medidos en campo y laboratorio y comprenden: abrasión, articulación, disolución, fragmentación, orientación, tamaño, bioerosión e incrustación. La definición de tafofacies fue tomada de Speyer y Brett (1986).

\section{Resultados}

\subsection{Litofacies (Figura 3)}

Las litologías principales identificadas en el análisis litofacial son las siguientes: S (Areniscas); L (Limolitas);

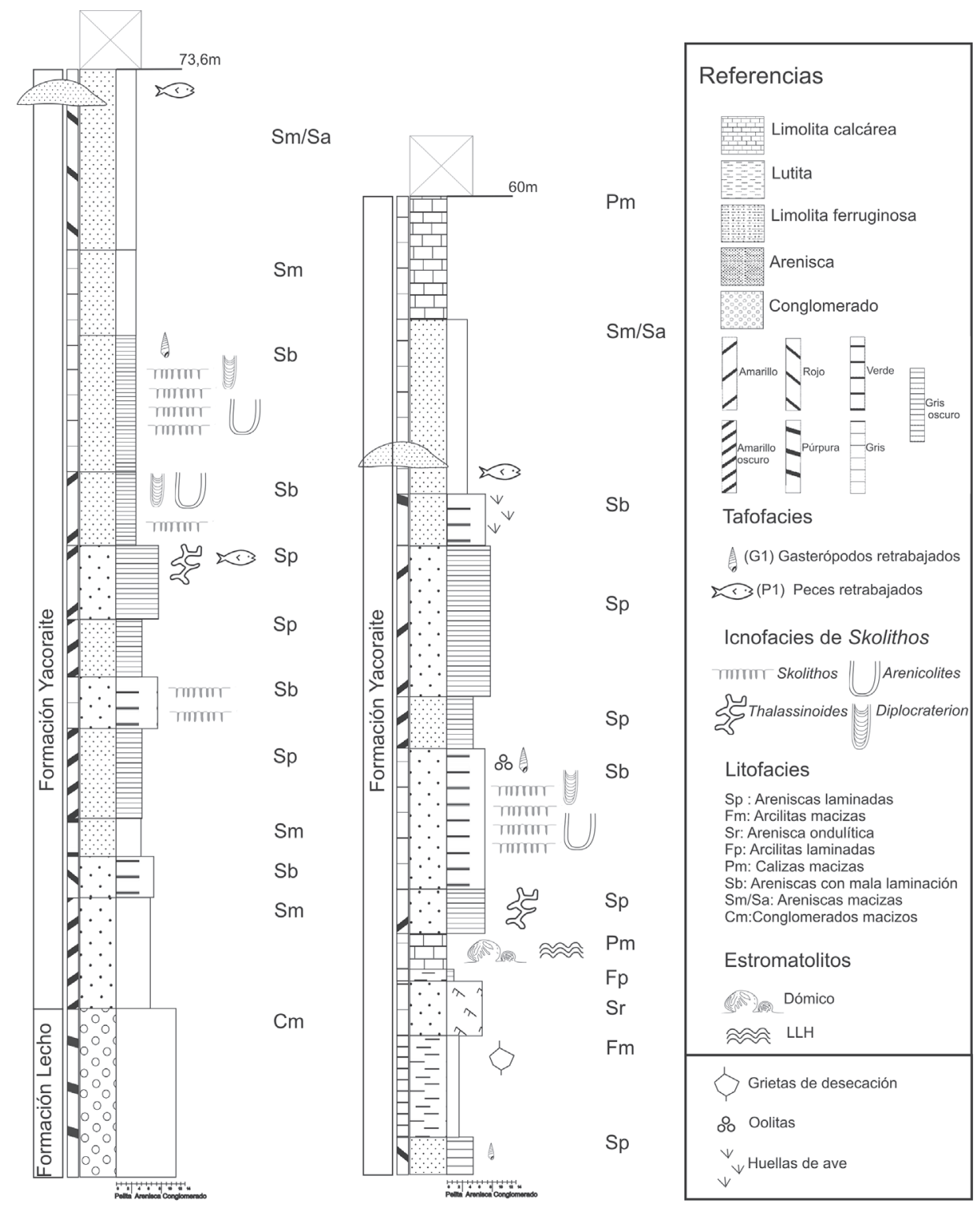

Figura 2: Perfiles estratigráficos de la Formación Yacoraite con indicación de litofacies, tafofacies e icnofacies aflorantes. 

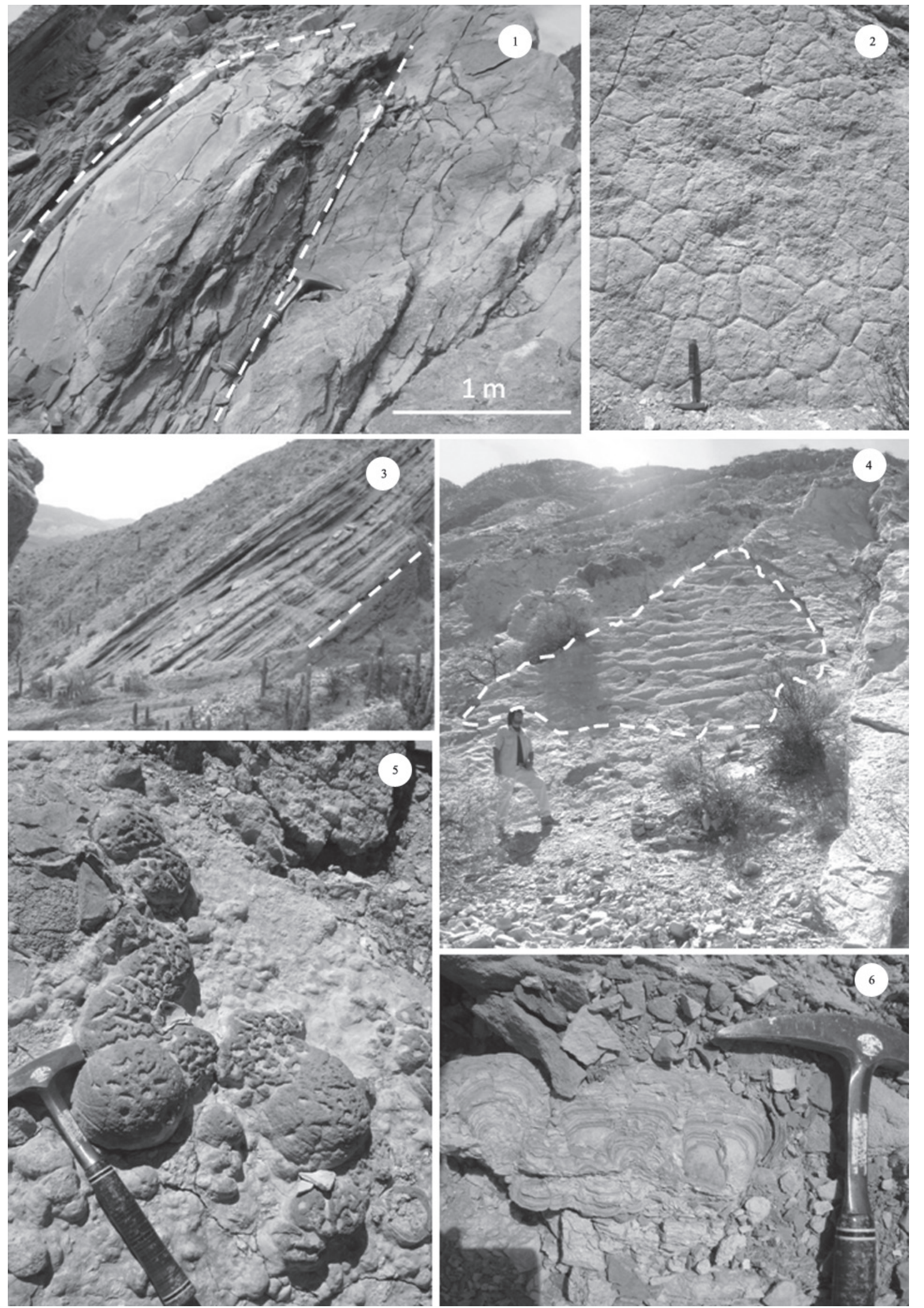

Figura 3: Litofacies de la Formación Yacoraite. 1- Litofacies Sm/Sa exhibiendo cuerpos monticulares tipo hummoky. 2- Grietas de desecación pertenecientes a la litofacies Fm. 3- Contacto entre las Formaciones Lecho y Yacoraite, dado por el contacto entre los conglomerados de la litofacies Cm (debajo de la línea punteada) y la litofacies Sm. 4- Óndulas simétricas de la litofacies Sr. 5- Estromatolitos dómicos pertenecientes a la litofacies Pm. 6- Estromatolitos LLH de la litofacies Pm 
Tabla 1: Litofacies principales de la Formación Yacoraite.

\begin{tabular}{|c|c|c|c|c|}
\hline $\begin{array}{l}\text { Asociación de } \\
\text { Litofacies }\end{array}$ & $\begin{array}{c}\text { Litología, paleontología e } \\
\text { icnofacies }\end{array}$ & $\begin{array}{l}\text { Forma y espesor de } \\
\text { los bancos }\end{array}$ & Estructura & Proceso sedimentario \\
\hline $\begin{array}{l}\text { Sp : Areniscas } \\
\text { laminadas }\end{array}$ & $\begin{array}{l}\text { Areniscas carbonáticas rojas } \\
\text { y amarillas finas a gruesas, } \\
\text { restos de gasterópodos, } \\
\text { peces mal preservados e } \\
\text { icnogénero Thalassinoides }\end{array}$ & $\begin{array}{l}\text { Bancos tabulares, } \\
\text { contactos de base y } \\
\text { techo netos, espesor } \\
\text { variable, alcanzando } \\
\text { los } 12 \text { m como } \\
\text { máximo }\end{array}$ & $\begin{array}{l}\text { Laminación } \\
\text { paralela }\end{array}$ & $\begin{array}{l}\text { Migración de lecho plano } \\
\text { por régimen de flujo } \\
\text { superior }\end{array}$ \\
\hline $\begin{array}{l}\text { Fm: Arcilitas } \\
\text { macizas }\end{array}$ & $\begin{array}{l}\text { Arcilitas grises oscuras } \\
\text { carbonáticas }\end{array}$ & $\begin{array}{l}\text { Bancos tabulares, } \\
\text { contactos de base y } \\
\text { techo netos, espesor } \\
\text { máximo } 10 \mathrm{~m}\end{array}$ & $\begin{array}{l}\text { Estructura } \\
\text { maciza, hacia el } \\
\text { techo grietas de } \\
\text { desecación }\end{array}$ & $\begin{array}{l}\text { Decantación o transporte } \\
\text { de tracción débil, seguido } \\
\text { por desecación y } \\
\text { contracción }\end{array}$ \\
\hline $\begin{array}{l}\text { Sr: Arenisca } \\
\text { ondulítica }\end{array}$ & $\begin{array}{l}\text { Areniscas medias con } \\
\text { cemento carbonático, grises } \\
\text { oscuras, con trenes muy } \\
\text { bien desarrollados de } \\
\text { óndulas de oscilación } \\
\text { (simétricas) con una } \\
\text { longitud de onda entre } 12 \\
\text { y } 20 \mathrm{~cm}\end{array}$ & $\begin{array}{l}\text { Bancos tabulares, } \\
\text { contactos de base y } \\
\text { techo netos, } 4 \mathrm{~m} \text { de } \\
\text { espesor máximo }\end{array}$ & $\begin{array}{l}\text { Estructura } \\
\text { ondulítica } \\
\text { simétrica por } \\
\text { oleaje }\end{array}$ & $\begin{array}{l}\text { Migración de óndulas de } \\
\text { corriente por régimen de } \\
\text { flujo inferior bidireccional }\end{array}$ \\
\hline $\begin{array}{l}\text { Fp: Arcilitas } \\
\text { laminadas }\end{array}$ & $\begin{array}{l}\text { Arcilitas carbonáticas grises } \\
\text { claras }\end{array}$ & $\begin{array}{l}\text { Bancos tabulares, } \\
\text { contactos de base y } \\
\text { techo netos, } 0.7 \mathrm{~m} \text { de } \\
\text { espesor máximo }\end{array}$ & $\begin{array}{l}\text { Laminación } \\
\text { paralela }\end{array}$ & Decantación suspensiva \\
\hline $\begin{array}{l}\text { Pm: Calizas } \\
\text { macizas }\end{array}$ & $\begin{array}{l}\text { Dolomías grises claras, } \\
\text { presencia de estromatolitos } \\
\text { dómicos y LLH } \\
\text { (hemiesferoides lateralmente } \\
\text { unidos) }\end{array}$ & $\begin{array}{l}\text { Bancos tabulares, } \\
\text { contactos de base y } \\
\text { techo netos, } 8 \mathrm{~m} \text { de } \\
\text { espesor máximo }\end{array}$ & $\begin{array}{l}\text { Estructura } \\
\text { maciza }\end{array}$ & $\begin{array}{l}\text { Alta productividad de } \\
\text { carbonato, poco aporte } \\
\text { detrítico y buena } \\
\text { disponibilidad de espacio } \\
\text { de acomodación e } \\
\text { inducción bioquímica de } \\
\text { carbonato provocada por } \\
\text { la actividad de algas } \\
\text { verdes y azules }\end{array}$ \\
\hline $\begin{array}{l}\text { Sb: Areniscas } \\
\text { con mala } \\
\text { laminación }\end{array}$ & $\begin{array}{l}\text { Arenisca carbonática fina a } \\
\text { media color gris claro, con } \\
\text { niveles oolíticos } \\
\text { intercalados, restos de } \\
\text { gasterópodos, huellas de ave } \\
\text { y abundantes trazas fósiles } \\
\text { pertenecientes a la } \\
\text { icnofacies de Skolithos }\end{array}$ & $\begin{array}{l}\text { Bancos tabulares, } \\
\text { contactos de base y } \\
\text { techo netos, } 11 \mathrm{~m} \mathrm{de} \\
\text { espesor máximo }\end{array}$ & Mala laminación & $\begin{array}{l}\text { Proceso deposicional } \\
\text { original desconocido por } \\
\text { la destrucción de la } \\
\text { estructura sedimentaria } \\
\text { por bioturbación }\end{array}$ \\
\hline $\begin{array}{l}\mathbf{S m} / \mathbf{S a}: \\
\text { Areniscas } \\
\text { macizas }\end{array}$ & $\begin{array}{l}\text { Arenisca carbonática fina, } \\
\text { color gris claro maciza, } \\
\text { intercalada con cuerpos } \\
\text { monticulares tipo hummoky } \\
\text { (HCS), presentando restos } \\
\text { de peces (Pycnodontiformes } \\
\text { e indeterminados) }\end{array}$ & $\begin{array}{l}\text { Bancos tabulares y } \\
\text { lenticulares } 11 \mathrm{~m} \text { de } \\
\text { espesor máximo }\end{array}$ & $\begin{array}{l}\text { Intervalo macizo } \\
\text { y estratificación } \\
\text { cruzada } \\
\text { aborregada o } \\
\text { monticular } \\
\text { (hummoky) }\end{array}$ & $\begin{array}{l}\text { Flujos oscilatorios puros y } \\
\text { flujos combinados de } \\
\text { menor profundidad } \\
\text { producidos por eventos de } \\
\text { tormenta }\end{array}$ \\
\hline $\begin{array}{l}\text { Cm: } \\
\text { Conglomerados } \\
\text { macizos }\end{array}$ & $\begin{array}{l}\text { Conglomerado macizo } \\
\text { matriz soportante, } \\
\text { polimíctico, constituido por } \\
\text { clastos de metamorfitas y } \\
\text { sedimentitas }\end{array}$ & $\begin{array}{l}\text { Banco tabular de } 6 \mathrm{~m} \\
\text { de espesor }\end{array}$ & $\begin{array}{l}\text { Estructura } \\
\text { maciza }\end{array}$ & $\begin{array}{l}\text { Flujos primarios y } \\
\text { secundarios de alta } \\
\text { energía }\end{array}$ \\
\hline
\end{tabular}


F (Arcilitas); P (Calizas); C (Conglomerados). Los calificativos faciales estructurales utilizados son: $m$ (maciza); p (laminación paralela); b (mala laminación); i (Estratificación inclinada); r (ondulitas); h (cruzada aborregada o hummocky). En la Tabla 1 se brinda una breve descripción de las litofacies identificadas.

\subsection{Tafofacies (Figura 4)}

Los conjuntos de rocas sedimentarias caracterizados por una particular, y a veces única, combinación de características preservacionales pueden ser designados como facies tafonómicas o tafofacies (Speyer y Brett, 1986). Para la interpretación y discusión de las tafofacies hay tres criterios importantes que deben ser tenidos en cuenta: tasa de sedimentación, oxigenación del medio sedimentario y energía del ambiente generador de cada tafofacies (Brett y Baird, 1986). En la Tabla 2 se presenta brevemente la interpretación de estos criterios para las tafofacies definidas, y a continuación una descripción de las tafofacies identificadas.

\subsubsection{G1: Gasterópodos retrabajados}

Descripción. Corresponde a una concentración monotípica, poliespecífica, dispersa, de gasterópodos pertenecientes a las litofacies Sb y Sp. Se observa una mala laminación asociada, probablemente por la acción de la bioturbación que eliminó la estructura sedimentaria original. La única especie identificada corresponde a Zygopleura maimarensis (Bonarelli, 1927).

Los índices tafonómicos principales son: fragmentación variable, siendo muy poco frecuente encontrar los peristomas preservados, el ápice siempre se presenta fragmentado. Se observa en todos los casos atrición de los bordes probablemente por transporte (abrasión mecánica), encontrándose redondeados los elementos ornamentales de la teleconcha. No se observa incrustación o bioerosión, y algunas porciones de la teleconcha presentan disolución química. Los gasterópodos se encuentran rellenos de $\mathrm{CaCO}_{3}$. No hay una orientación preferencial de las conchillas. No existe selección de tamaño para los gasterópodos, siendo polimodal su distribución.

Interpretación. La preservación de los gasterópodos es variable. La atrición de los bordes y ornamentaciones, el relleno carbonático y su baja selección de tamaño indican un tiempo prolongado de permanencia en la zona tafonómicamente activa (TAZ), aunque los bajos índices de atrición y fragmentación sugieren que el transporte lateral no fue importante. El relleno de los gasterópodos es del mismo material que la roca hospedante, lo que sugiere por lo menos un ciclo de exhumación y enterramiento, además de un entorno diagenético básico, lo que posibilitó una rápida formación de relleno carbonático, que permitió mantener la estructura de las conchillas evitando su colapso por presión diferencial. No es posible distinguir mezcla espacial o temporal. En relación a los atributos o índices tafonómicos
Tabla 2: Estimación de la tasa de sedimentación, oxigenación del medio sedimentario y energía del ambiente generador de cada tafofacies (Brett y Baird, 1986).

\begin{tabular}{|c|c|c|c|c|}
\hline Tafofacies & $\begin{array}{c}\text { Tasa de } \\
\text { sedimentación }\end{array}$ & $\begin{array}{c}\text { Oxigenación del } \\
\text { medio sedimentario }\end{array}$ & $\begin{array}{c}\text { Energía del } \\
\text { ambiente }\end{array}$ & $\begin{array}{l}\text { Profundidad } \\
\text { de formación }\end{array}$ \\
\hline $\begin{array}{l}\text { Gasterópodos } \\
\text { retrabajados } \\
\text { (G1) }\end{array}$ & Baja & Aeróbico & Alta & Baja \\
\hline $\begin{array}{l}\text { Peces } \\
\text { retrabajados } \\
(\mathrm{P} 1)\end{array}$ & Baja & Aeróbico & Alta & Baja \\
\hline
\end{tabular}

el grado de fragmentación de los gasterópodos es bajo, lo cual puede indicar que tuvieron retrabajos episódicos (Brett y Baird, 1986). La abrasión mecánica observada en la superficie de las conchillas puede ser atribuida a la acción del transporte por oleaje (Brett y Baird, 1986; Chave, 1964; Driscoll y Weltin, 1973). El grado parcial de disolución química de algunas porciones de la teleconcha puede deberse a pequeñas variaciones locales del $\mathrm{pH}$ en etapas diagenéticas posteriores al enterramiento definitivo.

\subsubsection{P1: Peces retrabajados}

Se compone de restos de peces Pycnodontiformes (Osteichthyes, Actinopterygii, Neopterygii) y Semionotiformes? (Osteichthyes, Actinopterygii). Es una asociación monotípica y poliespecífica. Los restos están integrados por dientes, fragmentos mandibulares, craneales y vértebras. Es muy importante el grado de desarticulación y corrosión de los mismos. Esta tafofacies se encuentra incluida en las litofacies $\mathrm{Sm} / \mathrm{Sa}$.

Interpretación. Los atributos tafonómicos más importantes observados son desarticulación y corrosión. No se observa selección hidrodinámica de partes o tamaño preferencial de bioclasto, por lo que la distribución de tamaño es polimodal. La desarticulación se debe en primera medida a un ambiente oxigenado que ocasionó el decaimiento de la materia orgánica que sustenta las carcasas y en segunda medida retrabajo por oleaje, por lo que se infiere mucho tiempo en la zona tafonómicamente activa (TAZ), habiéndose producido por lo menos un evento de exhumación y enterramiento, debido probablemente a los eventos de tormenta que los depositaron en tempestitas proximales. No se observa mezcla espacial, ni ambiental a partir de la fauna preservada.

\subsection{Icnofacies (Figura 5)}

Las asociaciones icnológicas principales observadas son:

\subsubsection{Asociación de Skolithos, icnofábrica del tipo "piperock"}

Interpretación. Densas asociaciones de Skolithos producen una icnofábrica frecuentemente denominada "piperock". Esta es una icnofábrica monoicnoespecífica, en la mayoría de los casos identificada en la icnofacies 


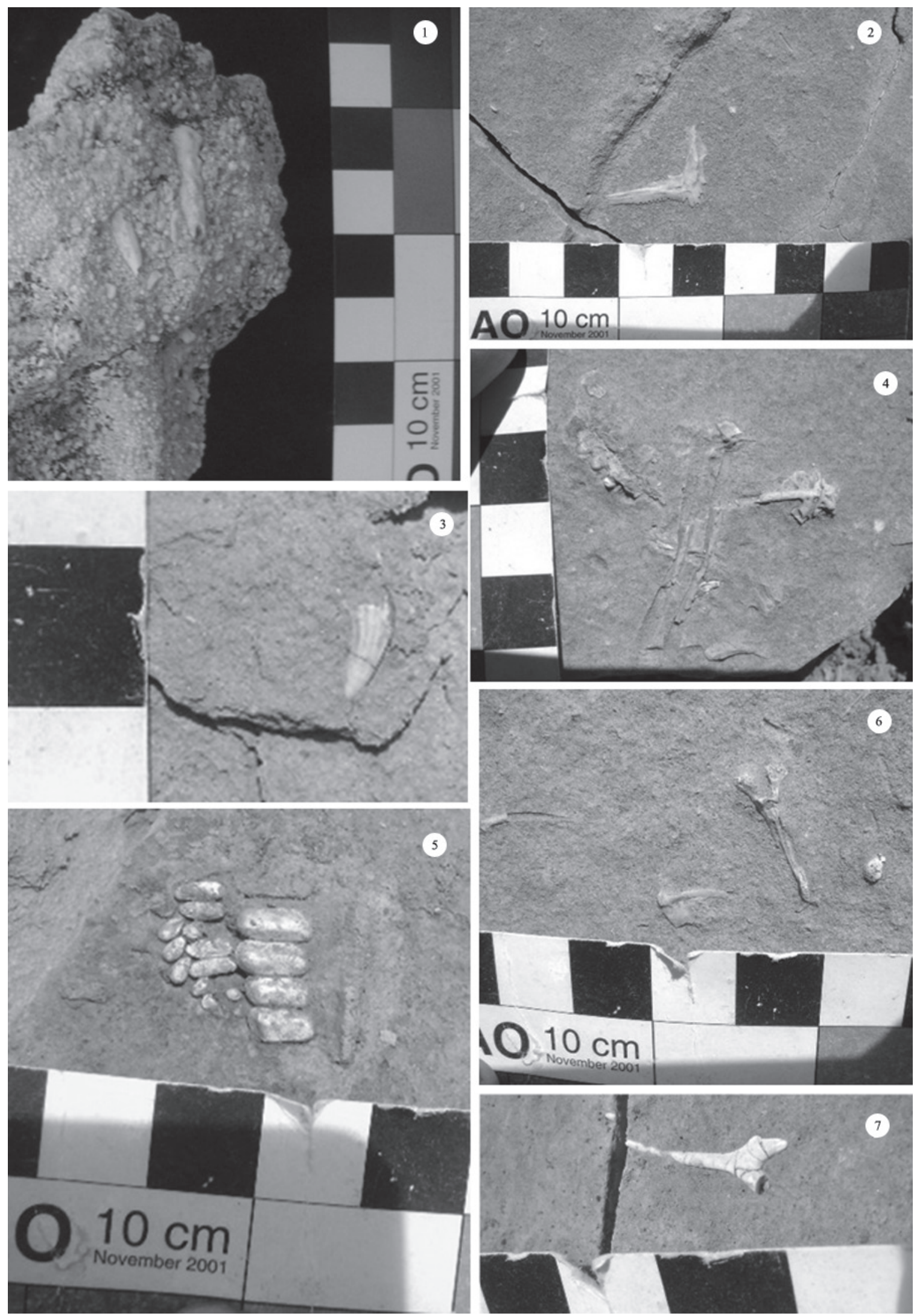

Figura 4: Tafofacies de la Formación Yacoraite. 1- Muestra de mano perteneciente a la tafofacies G1, con un ejemplar de Zygopleura maimarensis (Bonarelli, 1927). 2- Fragmento óseo de pez indeterminado correspondiente a la tafofacies P1. 3- Diente de pez Semionotiforme? de la tafofacies P1. 4- Restos de pez Pycnodontiforme de la tafofacies P1. 5- Dentición de pez Pycnodontiforme aislada, perteneciente a la tafofacies P1. 6- y 7- Fragmentos desarticulados de pez de la tafofacies P1. 


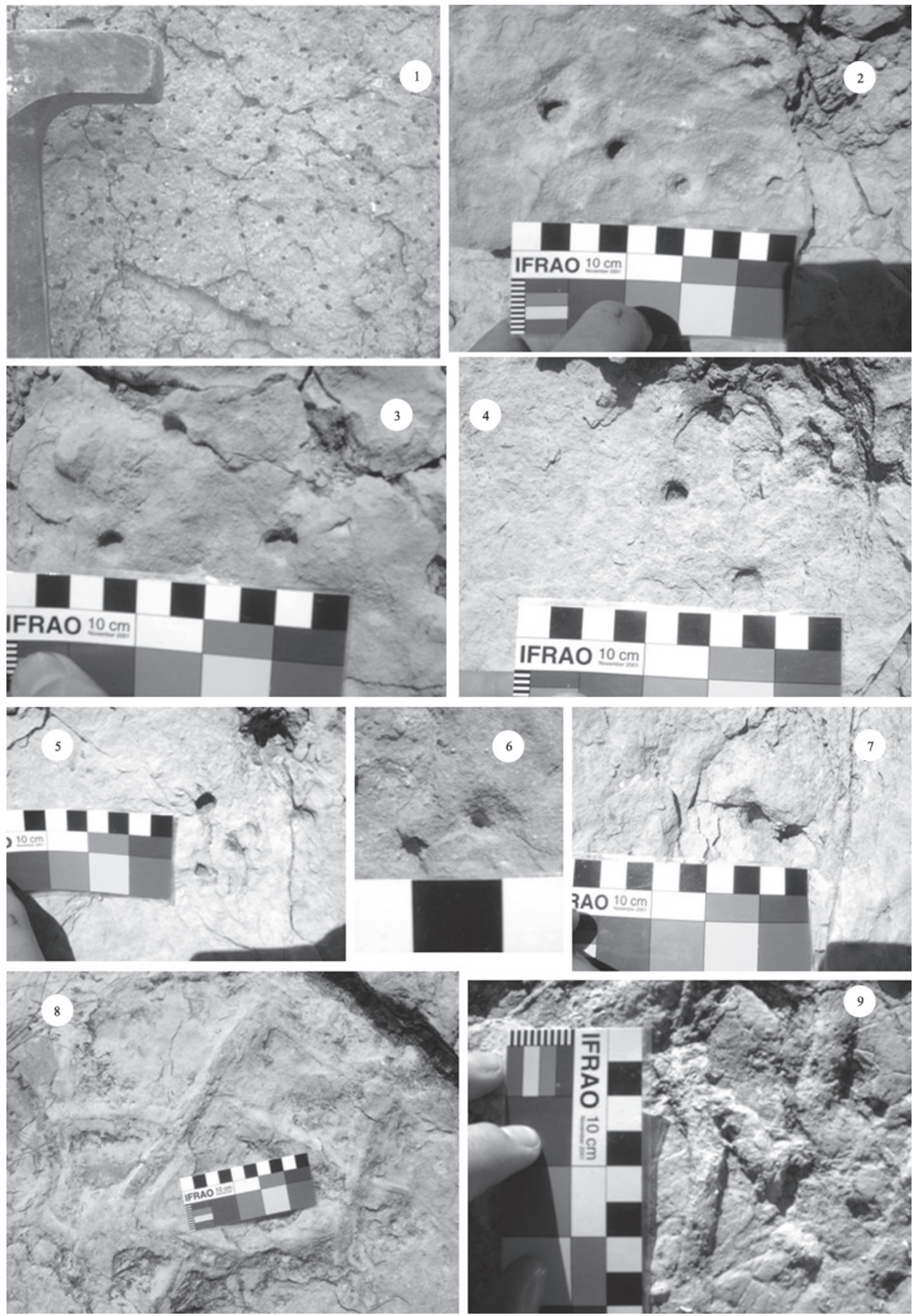

Figura 5: Icnofacies de Skolithos. 1- Roca exhibiendo Skolithos isp. y presentando bioturbación del tipo pipe rock. 2-, 3- y 4- Arenicolites isp. 5-, 6- y 7- Diplocraterion isp. 8- y 9- Thalassinoides isp. 
de Skolithos. Es normalmente encontrada en areniscas formadas en facies marinas poco profundas, siendo propia de zonas litorales arenosas de gran energía (Droser, 1991).

\subsubsection{Asociación de Skolithos, Arenicolites y Diplocraterion}

Interpretación. Skolithos puede ser encontrado en una variedad de ambientes deposicionales, desde marinos a continentales, habiendo sido ubicados en canales de marea, llanuras de marea, deltas, estuarios y depósitos de tormenta (Droser, 1991). Por su parte Diplocraterion y Arenicolites han sido caracterizados como icnogéneros propios de ambientes de alta energía (Bromley y Asgaard, 1979; Fürsich, 1975). En consecuencia consideramos a esta icnocenosis como típica de zonas de alta energía.

\subsubsection{Asociación de Thalassinoides}

Interpretación. Thalassinoides es un icnogénero del tipo cross-facies trace fossil, es decir, que puede ser encontrado en las facies más diversas (Frey et al., 1978). El establecimiento de esta asociación indica por lo menos un ambiente con buena disponibilidad de oxígeno (Verde, 2002).

Estas icnoasociaciones pueden ser asignadas a la icnofacies arquetípica de Skolithos, generada probablemente en un ámbito marino mixto, de baja profundidad y alta energía originada por corrientes u olas. Representa el litoral inferior a infralitoral, donde se depositan sedimentos limosos a limpios, bien seleccionados, propensos a erosión y depositación abrupta (Frey y Pemberton, 1984).

\section{Discusión y consideraciones paleoambientales}

La tafofacies de gasterópodos retrabajados sobre calizas oolíticas indica proximidad a la costa y alta energía por acción del oleaje. En relación a la fidelidad temporal de la concentración, hay que considerar que se ha demostrado a través de estudios actuotafonómicos en moluscos que puede haber una diferencia de miles de años entre organismos que se encuentran uno junto a otro en un mismo estrato (Flessa y Kowalewski et al., 1994; Kowalewski et al., 1998), siendo habitual en este tipo de ambientes y esperable en función de la hipótesis de por lo menos un ciclo de enterramiento y exhumación. De acuerdo a la clasificación bioestratinómica de las concentraciones fosilíferas propuesta por Kidwell et al. (1986), estos atributos tafonómicos caracterizan a un conjunto de tipo sedimentológico, que corresponde a una acumulación de partes duras durante un periodo de sedimentación neta baja que origina la acumulación de especímenes autóctonos/parautóctonos, que han sido retrabajados en cierto grado pero sin ser transportados fuera de su hábitat original.

La preservación de tafofacies de peces retrabajados en estructuras sedimentarias con estratificación entrecruzada tipo monticular (hummoky), exhibiendo parte de las carcasas multielementales de peces, indicaría la acción de tempestitas proximales (Brett y Baird, 1986) y constituiría una concentración del tipo sedimentológico (Kidwell et al., 1986). Sobre estas concentraciones podemos decir, en términos de su ciclicidad tafonómica, que pertenecen a secuencias tafonómicas elementales cuya duración es extremadamente breve (minutos), correspondiente con sedimentación de eventos o tempestitas ocurridos durante tormentas, y que pueden caracterizar eventos de mortandad masiva, sobre ambientes de plataformas epicontinentales carbonáticas (Fernández-López, 1999), siendo consecuente con la caracterización sedimentológica del depósito.

La ausencia de una fauna marina típica, como así también la dominancia de la icnofacies de Skolithos empobrecida, sugiere una columna de agua salobre de pocos metros que estaría controlando la baja diversidad de icnogéneros, siendo indicadora de condiciones de alta a moderada energía (Buatois et al., 2002; Frey y Pemberton 1984; Pemberton et al., 1992). Los principales controles para la distribución de icnofacies serían el tipo de sustrato, la energía del medio, el contenido de nutrientes, la oxigenación, la salinidad y las tasas de sedimentación y erosión (Buatois et al., 2002), por lo que se debe contemplar más de una línea de análisis a la hora de realizar inferencias paleobatimétricas. Esta situación es discutida extensamente por muchos autores (Byers, 1982; Ekdale, 1988; Frey et al., 1990). El icnogénero Skolithos en ambientes marinos es considerado principalmente como una estructura domichnial realizada por forónidos o anélidos (Sokolosky, 2005), quienes a partir de esta estrategia se protegerían de las condiciones del medio y, en el caso de la icnofábrica del tipo pipe rock, indicaría zonas litorales arenosas de gran energía (Bottjer y Dorser, 1991). Es interesante destacar que la presencia de estromatolitos de los tipos LLH y dómicos sugiere por lo menos un entorno de poca profundidad, con buena disponibilidad de $\mathrm{CaCO}_{3}$ disuelto, desarrollándose estas estructuras en un ambiente de planicies de mareas (Logan et al., 1964), ya sea en sectores supramareales o en sectores intermareales superiores.

\section{Conclusiones}

Las características paleoambientales de cuenca carbonática somera y extendida de la Formación Yacoraite (Marquillas, 1985) han permitido la preservación de un gran registro paleontológico, susceptible de ser correlacionable. En este sentido, el avance en el estudio tafonómico de estas concentraciones y de las estructuras sedimentarias biogénicas asociadas es coincidente con la sedimentología del depósito, y puede brindar respuestas geológicas interesantes para aquellas variaciones ambientales de menor escala.

Los resultados de la caracterización tafofacial, icnofacial y el análisis de las litofacies sedimentarias permiten la ubicación de un ambiente litoral, salobre, somero, con buena oxigenación, alta energía y buena disponibilidad 
de nutrientes, dominado por el oleaje y sedimentación episódica breve producto de tormentas.

\section{Agradecimientos}

Agradecemos al CONICET por la financiación del proyecto. Queremos también expresar reconocimiento a los Dres. Guillermo Aceñolaza, Sergio Georgieff y Silvana Urquiza por su colaboración. A Pablo Machuca Arias y Franco Campos por la ayuda en las tareas de campo. Nuestro especial agradecimiento para los Dres. Ricardo N. Alonso y Sixto R. Fernández-López, quienes con sus valiosos comentarios mejoraron el manuscrito original.

\section{Referencias}

Alonso, R.N., 1980, Dinosaurs traces (Ornithopoda, Hadrosauridae) from Upper Cretaceous of Argentina: Acta Geológica Lilloana, 15, 55-63.

Alonso, R.N., 2007, Dinosaurios salteños y argentinos: un fascinante capítulo en la historia de la Tierra: Salta, Argentina, Crisol EdicionesUNSa-CONICET, $180 \mathrm{p}$.

Alonso, R.N., Marquillas, R.A., 1986, New site with dinosaurs tracks, and first record of bird tracks from Yacoraite Formation (Maastrichtian) in the north argentinian, en Actas $4^{\circ}$ Congreso Argentino de Paleontología y Bioestratigrafía: Mendoza, Argentina, Asociación Paleontológica Argentina, 33-41.

Arratia, G., Cione, A.L., 1996, The fish fossil record of Southern South America: Münchener Geowissenschaft Abhanlungen, 30, 9-72.

Bender Kotzian, C., Simoes, M.G., 2006, Taphonomy of recent freshwater molluscan death assemblages, Touro Passo stream, southern Brazil: Revista Brasileira de Paleontología, 9, 243-260.

Bonarelli, G., 1927, Fósiles de la Formación Petrolífera: Boletín de la Academia Nacional de Ciencias de Córdoba, 30, 55-115.

Bottjer, D.J., Dorser, M.L., 1991, Ichnofabric and basin analysis: Palaios, 6, 199-205.

Bressan, G.S., Palma, R.M., 2008, Tafonomía e icnología de los depósitos de tormenta de la Formación Bardas Blancas (Jurásico InferiorMedio), Mendoza, Argentina: Ameghiniana, 45, 513-528.

Brett, C.E., Baird, G.C., 1986, Comparative taphonomy; a key to paleoenvironmental interpretation based on fossil preservation: Palaios, 1, 27-227.

Bromley, R.G., 1990, Trace fossils. Biology and taphonomy: London, Unwin Hyman, $310 \mathrm{p}$.

Bromley, R.G, 1996, Trace fossils. Biology, taphonomy and applications: London, Chapman and Hall, $361 \mathrm{p}$.

Bromley, R., Asgaard, U., 1979, Triassic freshwater ichnocoenoses from Carlsberg Fjord, East Greenland: Palaeogeography, Palaeoclimatology, Palaeoecology, 28, 39-90.

Buatois, L.A., Mángano, M.G., Aceñolaza, F.G., 2002, Trazas fósiles: Señales de comportamiento en el registro estratigráfico: Trelew, Chubut, Argentina, Museo Paleontológico Egidio Feruglio, 382 p.

Byers, C.W., 1982, Geological significance of marine biogenic sedimentary structures, in McCall, P.L., Tevesz, M.J.S. (eds.), Animal-sediment Relations: the Biogenic Alteration of Sediments: New York, Plenum, 221-256.

Chave, K.E., 1964, Skeletal durability and preservation, in Imbrie, J., Newell, N. (eds.), Approaches to Paleoecology: New York, John Wiley and Sons, 377-387.

Cione, A.L., 1977, Algunas consideraciones sobre Pycnodontiformes (Pisces, Holostei) procedentes de la Formación Yacoraite, Cretácico Tardío de la Provincia de Salta, Argentina: Ameghiniana,14, 315316.
Cione, A.L., Pereira., S.M., 1985, Los peces de la Formación Yacoraite (Cretácico Tardío-Terciario?, Noroeste argentino) como indicadores de salinidad: Revista de la Asociación Geológica Argentina, 40, 83-88.

Cione, A.L., Pereira, S.M., Alonso, R., Arias, J., 1985, Los bagres (Osteichthyes-Siluriformes) de la Formación Yacoraite (Cretácico Tardío): Consideraciones biogeográficas y bioestratigráficas: Ameghiniana, 21, 294-304.

Cónsole Gonella, C.A, Aceñolaza, F.G., 2008, Estudio taxonómico y tafonómico sobre fauna de gasterópodos de la Formación Yacoraite en la localidad de Jueya, Provincia de Jujuy, Argentina (resumen), en XVII Congreso Geológico Argentino: San Salvador de Jujuy, Jujuy, Argentina, Asociación Geológica Argentina, 5, 1487.

Cónsole Gonella, C.A., Griffin, M., Aceñolaza, F.G., 2009, Gastropods associated with Fossil Traces from Yacoraite Formation (Maastrichtian-Danian), and its paleoenvironmental significance, Jujuy, Northwestern Argentina: Acta Geologica Sinica, 83: 860-867.

Davies, N.S., Sansom, I.J., Albanesi, G.L., Cespedes, R, 2007, Ichnology, palaeoecology and taphonomy of a Gondwanan early vertebrate habitat: Insights from the Ordovician Anzaldo Formation, Bolivia: Palaeogeography, Palaeoclimatology, Palaeoecology, 249, 18-35.

Donovan, S.K., Jagt, J.W.M., 2004, Taphonomic and ethologic aspects of the ichnology of the Maastrichtian of the type area (Upper Cretaceous, The Netherlands and Belgium): Bulletin de l'Institut Royal des Sciences Naturelles de Belgique, Sciences de la Terre, 74, 119-127.

Driscoll, E.G., Weltin, T.P., 1973, Sedimentary parameters as factors in abrasive shell reduction: Palaeogeography, Palaeoclimatology, Palaeoecology, 13, 275-288.

Droser, M.L., 1991, Ichnofabric of the Paleozoic Skolithos ichnofacies and the nature and distribution of Skolithos piperock: Palaios, 6, 316-325.

Ekdale, A.A, 1988, Pitfalls of paleobathymetric interpretations based on trace fossils assemblages: Palaios, 3, 464-472.

Fernandez-López, S.R., 1999, Applied palaeontology and sequence stratigraphy in carbonate epicontinental platforms, in Rocha, R.B., Silva, C.M., Caetano, P.S., Kullberg., J.C. (eds.), Links between fossils assemblages and sedimentary cycles and sequences: Lisboa, European Palaeontological Association, 9-13.

Flessa, K.W., Kowalewski, M., 1994, Shell survival and time-averaging in near shore and shelf environments: estimates from the radiocarbon literature: Lethaia, 27, 153-165.

Frey, R.W., Pemberton, S.G., 1984, Trace fossil facies models, in Walker, R.G. (ed.), Facies models: Ottawa, Canada, Geoscience Canada, 189-207.

Frey, R.W., Seilacher, A., 1980, Uniformity in marine invertebrate ichnology: Lethaia, 13, 183-207.

Frey, R.W., Howard, J.D., Pryor, W.A., 1978, Ophiomorpha: its morphologic, taxonomic, and environmental significance: Palaeogeography Palaeoclimatology Palaeoecology, 23, 199-229.

Frey, R.W., Pemberton, S.G., Saunders., T.D.A., 1990, Ichnofacies and Bathymetry: A Passive Relationship: Journal of Paleontology, 64, 155-158.

Fürsich, F.T., 1975, Trace fossils as environmental indicators in the Corallian of England and Normandy: Lethaia, 8, 151-172.

Gasparini, Z.B., Buffetaut, E., 1980, Dolichochampsa minima, n.g. n.sp., a representative of a new family of eusuchian crocodiles from the Late Cretaceous of northern Argentina: Neues Jahrbuch für Geologie und Paläontologie Monatshefte, 8, 257-271.

Kidwell, S.M., Fuersich, F.T., Aigner, T., 1986, Conceptual framework for the analysis and classification of fossil concentrations: Palaios, $1,228-238$.

Kietzmann, D.A., Palma, R.M., 2009, Tafofacies y biofacies de Formación Vaca Muerta en el sector surmendocino de la Cuenca Neuquina: implicancias paleoecológicas, sedimentológicas y estratigráficas: Ameghiniana, 46, 321-343.

Kowalewski, M., Flessa, K.W., Aggen, J.A., 1994, Taphofacies analysis of recent shelly cheniers (beach ridges), northeastern Baja California, Mexico: Facies, 31, 209-241. 
Kowalewski, M., Goodfriend, G.A, Flessa, K.W, 1998, High-resolution estimates of temporal mixing within shell beds: the evils and virtues of time averaging: Paleobiology, 24, 287-304.

Lazo, D.G., 2006, Análisis tafonómico e inferencia del grado de mezcla temporal y espacial de la macrofauna del Miembro Pilmatué de la Formacion Agrio, Cretácico Inferior de cuenca Neuquina, Argentina: Ameghiniana, 43, 311-326.

Logan, B.W., Rezak, R., Ginsburg, R.N., 1964, Classification and environmental significance of algal stromatolites: Journal of Geology, 72, 68-83.

Marquillas, R.A., 1985, Estratigrafía, sedimentológica y paleoambientes de la Formación Yacoraite (Cretácico Superior) en el sector sur de la cuenca: Salta, Argentina, Universidad Nacional de Salta, Tesis doctoral, $139 \mathrm{p}$.

Marquillas, R.A., Salfity, J.A., 1994, Relaciones estratigráficas regionales de la Formación Yacoraite (Cretácico Superior) en el norte de Argentina, en VII Congreso Geológico Chileno: Concepción, Chile, Sociedad Geológica de Chile, 479-483.

Marquillas, R., Del Papa, C., Sabino, I., Heredia, J., 2003, Prospección del límite K/T en la cuenca del Noroeste, Argentina: Revista de la Asociación Geológica Argentina, 58, 271-274.

Marquillas R.A., Del Papa, C., Sabino, I.F., 2005, Sedimentary aspects and paleoenvironmental evolution of a rift basin: Salta Group (Cretaceous-Paleogene), northwestern Argentina: International Journal of Earth Sciences, 94, 94-113.

Marquillas R., Sabino, I., Nóbrega Sial, A., Del Papa, C., Ferreira, V., Matthews S., 2007, Carbon and oxygen isotopes of MaastrichtianDanian shallow marine carbonates: Yacoraite Formation, northwestern Argentina: Journal of South American Earth Sciences, 23, 304-320.

Moreno, J.A., 1970, Estratigrafía y paleogeografía del Cretácico Superior en la cuenca del noroeste argentino, con especial mención de los Subgrupos Balbuena y Santa Bárbara: Revista de la Asociación Geológica Argentina, 25, 9-44.
Parsons, K.M., Brett, C.E., 1991, Taphonomic processes and biases in modern marine environment, in Donovan, S.K. (ed.), The processes of fossilization: Columbia, Columbia University, 23-65.

Pemberton, S.G., MacEachern, J.A., Frey, R.W., 1992, Trace fossils facies models: Environmental and allostratigraphic significance, in Walker, R.G., James, N.P. (eds.), Facies models and sea level changes: Ottawa, Canada, Geological Association of Canada, 47-72.

Reyes, F.C., Salfity, J.A., 1973, Consideraciones sobre la estratigrafía del Cretácico (Subgrupo Pirgua) del noroeste argentino, en V Congreso Geológico Argentino: Buenos Aires, Argentina, Asociación Geológica Argentina, 355-385.

Sokolosky, W.P., 2005, Sedimentology and ichnology of late cambrian to early Ordovician skolithos sandstone in the deadwood formation, Northern black hills, South Dakota, and southeastern Bear lodge mountains, Wyoming: Toledo, Ohio, U.SA., The University of Toledo, Master Thesis, $220 \mathrm{p}$.

Speyer, S.E., Brett, C.E., 1986, Trilobite taphonomy and Middle Devonian taphofacies: Palaios, 1, 312-327.

Turner, J.C.M., 1959, Estratigrafía del cordón de Escaya y de la sierra de Rinconada (Jujuy): Revista de la Asociación Geológica Argentina, $13,15-39$.

Verde, M., 2002, Ichnology from Camacho Formation (Upper Miocene) of Uruguay: Montevideo, Uruguay, Universidad de la República, Tesis de maestría, $124 \mathrm{p}$.

Walker, C.A., 1981, New subclass of birds from the Cretaceous of South America: Nature, 292, 51-53.

Manuscrito recibido: Marzo 12, 2010.

Manuscrito corregido recibido: Mayo 14, 2010

Manuscrito aceptado: Mayo 30, 2010. 